\title{
Very small deletions within the NESP55 gene in pseudohypoparathyroidism type 1b
}

\author{
Faisal I Rezwan ${ }^{1,6}$, Rebecca L Poole ${ }^{1,2,6}$, Trine Prescott ${ }^{3}$, Joanna M Walker ${ }^{4}$, I Karen Temple T $^{1,5}$ \\ and Deborah JG Mackay ${ }^{\star 1,2}$
}

Pseudohypoparathyroidism (PHP) is caused by reduced expression of genes within the GNAS cluster, resulting in parathormone resistance. The cluster contains multiple imprinted transcripts, including the stimulatory G protein $\alpha$ subunit (Gs- $\alpha$ ) and NESP55 transcript preferentially expressed from the maternal allele, and the paternally expressed XLas, $A / B$ and antisense transcripts. PHP1b can be caused by loss of imprinting affecting GNAS A/B alone (associated with STX16 deletion), or the entire GNAS cluster (associated with deletions of NESP55 in a minority of cases). We performed targeted genomic nextgeneration sequencing (NGS) of the GNAS cluster to seek variants and indels underlying PHP1b. Seven patients were sequenced by hybridisation-based capture and fourteen more by long-range PCR and transposon-mediated insertion and sequencing. A bioinformatic pipeline was developed for variant and indel detection. In one family with two affected siblings, and in a second family with a single affected individual, we detected maternally inherited deletions of 40 and $33 \mathrm{bp}$, respectively, within the deletion previously reported in rare families with PHP1b. All three affected individuals presented with atypically severe PHP1b; interestingly, the unaffected mother in one family had the detected deletion on her maternally inherited allele. Targeted NGS can reveal sequence changes undetectable by current diagnostic methods. Identification of genetic mutations underlying epigenetic changes can facilitate accurate diagnosis and counselling, and potentially highlight genetic elements critical for normal imprint setting.

European Journal of Human Genetics (2015) 23, 494-499; doi:10.1038/ejhg.2014.133; published online 9 July 2014

\section{INTRODUCTION}

Pseudohypoparathyroidism (PHP) is caused by insensitivity to parathormone $(\mathrm{PTH})$, and the majority of cases are associated with insufficiency of the G protein stimulatory alpha subunit (Gs- $\alpha){ }^{1}$ In PHP type 1a (OMIM \#103580), additional features may include obesity, short stature, brachydactyly, ectopic ossification and cognitive impairment. PHP1b (OMIM \#603233) is classically defined as sharing the biochemical but not the skeletal features of PHPla, though this distinction is not absolute. ${ }^{2}$ PHP1b is not associated with coding mutations of GNAS, but with epigenetic errors altering its expression. The GNAS gene cluster (OMIM + 139320) on human chromosome 20 is under complex imprinted regulation, with transcripts variably expressed from the maternal allele, the paternal allele, or biallelically, in a tissue-dependent fashion. ${ }^{3}$ The clinical suspicion of PHP1b is confirmed by detecting abnormal DNA methylation at imprinting control regions within the GNAS cluster. ${ }^{4-6}$ Some patients show alterations at multiple imprinting control regions (ICRs) within the cluster, but the most consistent aberration is hypomethylation of the $\mathrm{A} / \mathrm{B}$ differentially methylated region directly upstream of the major coding isoform of GNAS. Isolated hypomethylation of this region is the commonest alteration in familial PHP1b; in all cases so far described, it has been associated with deletion of a presumed regulatory element within the STX16 gene. ${ }^{7,8}$ Much rarer are deletions affecting NESP55; ${ }^{9-11}$ these are accompanied by epigenetic disruption throughout the locus, and evidence from murine models suggests they disrupt transcription and thereby primary imprint setting throughout the GNAS cluster. ${ }^{12}$ However, apart from these and some cases of paternal uniparental disomy (UPD20pat), ${ }^{13}$ the majority of PHP1b cases seem to be caused by a primary epimutation without an underlying genetic aberration.

We hypothesised that additional genetic variants may result in epimutations of the GNAS differentially methylated regions and screened PHP1b patients, using genomic next-generation sequencing through the GNAS cluster.

\section{MATERIALS AND METHODS}

Patient recruitment: ethics

Clinical diagnosis of PHP1b was made locally by referring clinicians, and patients were consented into the research study 'Imprinting disorders-finding out why' (IDFOW: Southampton and South West Hampshire Research Ethics approval 07/H0502/85) through the UK Comprehensive Local Research network (www.southampton.ac.uk/geneticimprinting/informationpatients/ imprintingfindingoutwhy.page, accessed September 2013). Diagnosis was confirmed by methylation-specific PCR and microsatellite analysis as described. ${ }^{14}$ Twenty patients had PHP1b with methylation anomalies affecting the GNAS AB, XLAS, NESP-AS and NESP differentially methylated regions (DMRs), but no evidence of UPD20 or NESP deletion; 9 STX16 deletions were also excluded; and there was no evidence of

${ }^{1}$ Human Genetics and Genomic medicine, Faculty of Medicine, University of Southampton, Southampton, UK; ${ }^{2}$ Wessex Regional Genetics Laboratory, Salisbury District Hospital, Salisbury NHS Foundation Trust, Salisbury, UK; ${ }^{3}$ Department of Medical Genetics, Oslo University Hospital, Oslo, Norway; ${ }^{4}$ Queen Alexandra Hospital, Portsmouth, UK; ${ }^{5}$ Wessex Clinical Genetics Service, Princess Anne Hospital, University Hospital Southampton NHS Foundation Trust, Southampton, UK

*Correspondence: Dr DJG Mackay, Wessex Regional Genetics Laboratory, Salisbury District Hospital, Salisbury NHS Foundation Trust, Salisbury SP2 8BJ, UK Tel: + (044) 1722 425048; Fax: + (044) 1722 331531; E-mail: djgm@soton.ac.uk

${ }^{6}$ These authors contributed equally to this work.

Received 18 October 2013; revised 15 May 2014; accepted 30 May 2014; published online 9 July 2014 
hypomethylation at any other imprinted locus. Two control PHP1b patients were included: one with UPD20pat and one with a deletion of STX16.

Clinical findings in the two families with genetic variants are presented in Table 1. Briefly:

Family 1: a non-dysmorphic sister and brother presented with exerciseinduced muscle cramping in childhood and hypocalcemia. Skeletal survey was unremarkable. The mother was clinically unaffected.

Family 2: a male presented at 2 years 3 months with faltering growth, increasing weight and global developmental delay, a consistently raised serum phosphate and subsequently a raised serum PTH. Skeletal survey was unremarkable. Family history was non-contributory (Figure 1h).

\section{DNA methylation analysis}

Methylation status was initially determined by methylation-specific PCR, as described, ${ }^{14}$ and then more comprehensively assessed by methylation-specific pyrosequencing, as described ${ }^{5}$ in all patients, and compared with normal controls (Supplementary Table 1).

Table 1 Clinical features in the affected individuals in families 1 and 2

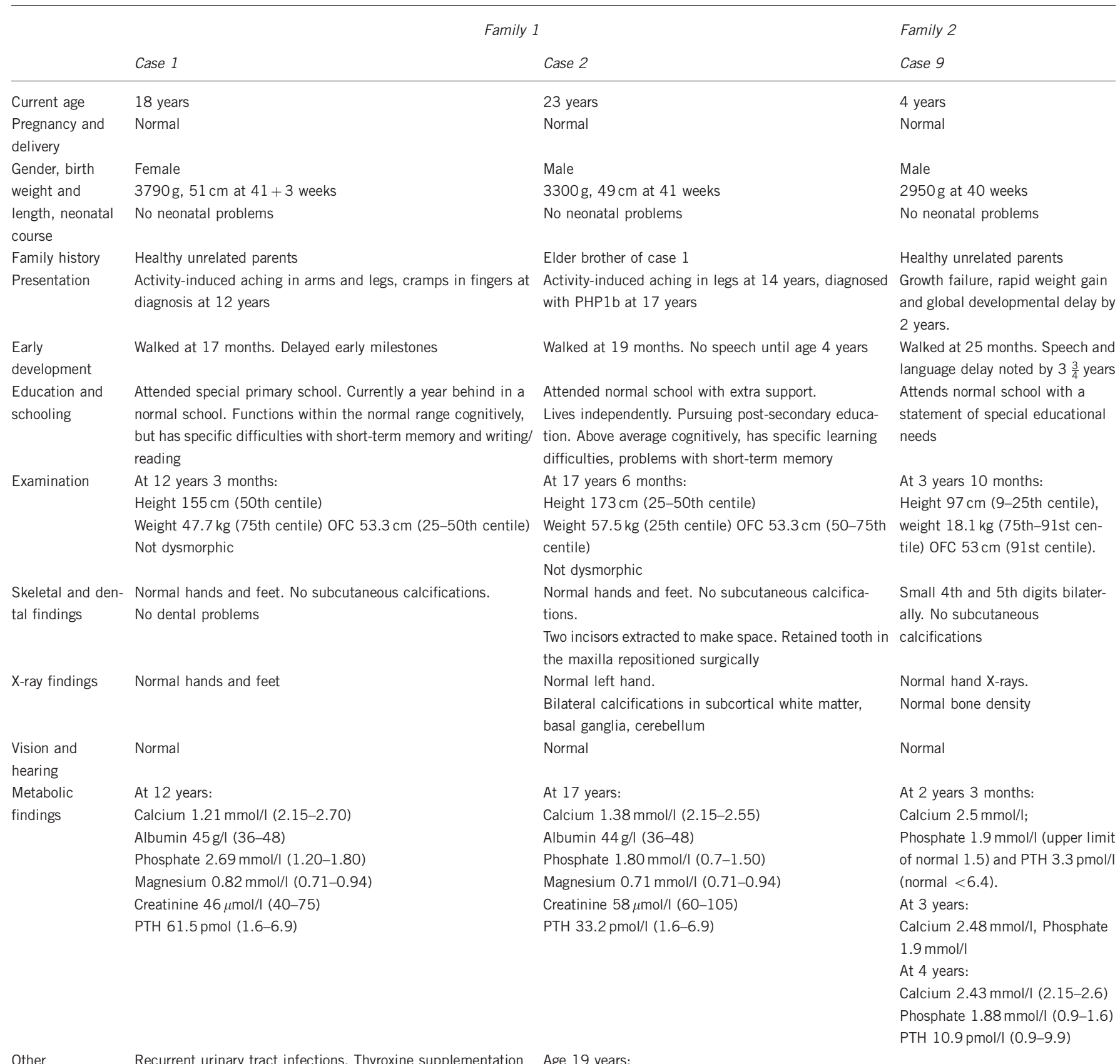

Other

Recurrent urinary tract infections. Thyroxine supplementation Age 19 years

from age 14 years for borderline low free T4 with normal TSH Hyperechogenic foci in both kidneys, possibly normal variant

GNAS Exons 2-13: no coding variants Exons 2-13: no coding variants

Exons 2-13: no coding variants 
Sequencing analysis

Targeted NGS. Genomic DNA was captured in the region chr20.GRCh37: g.57200800_57627000 using a targeted SureSelect array (Agilent Technologies UK Ltd, Wokingham, UK; $0.5-2.9 \mathrm{Mb}$ capture). The array design (Agilent SureDesign, Agilent Technologies UK Ltd) had baseline $\times 2$ coverage, moderate stringency, moderate masking of repeats and manual boosting in order to reduce replication of high GC-content baits. In total, $3 \mu \mathrm{g}$ DNA was sheared using a Bioruptor (Diagenode s.a., Seraing, Belgium) and then captured using the Agilent SureSelect system, according to the manufacturer's instructions. DNA samples were indexed, and 500 cycles of sequencing performed on an Illumina MiSeq (Reagent kit v2, Illumina UK, Chesterford, UK), generating $\sim 800000$ clusters, $89 \%$ passing filter and $7 \mathrm{~Gb}$ data. Further

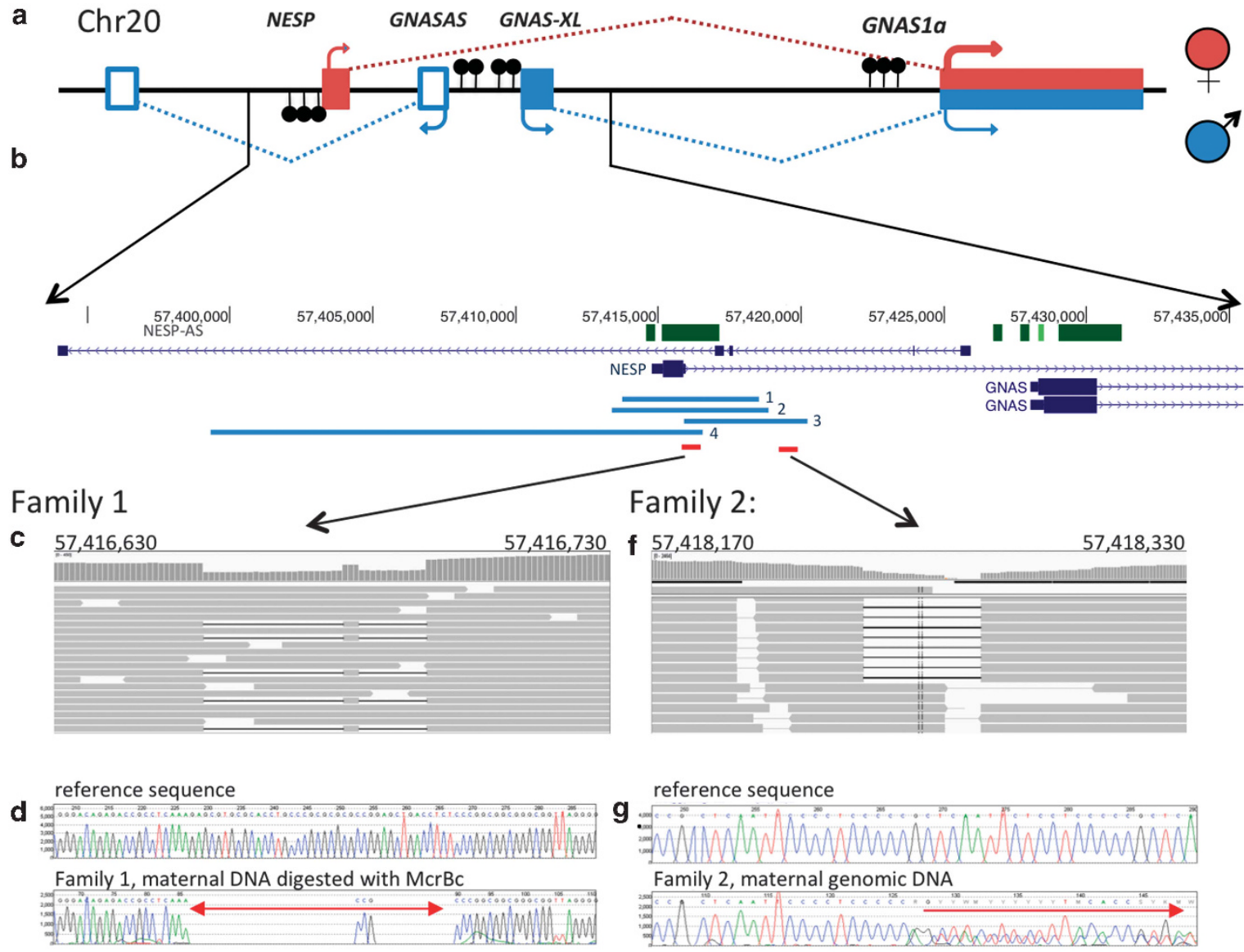

e
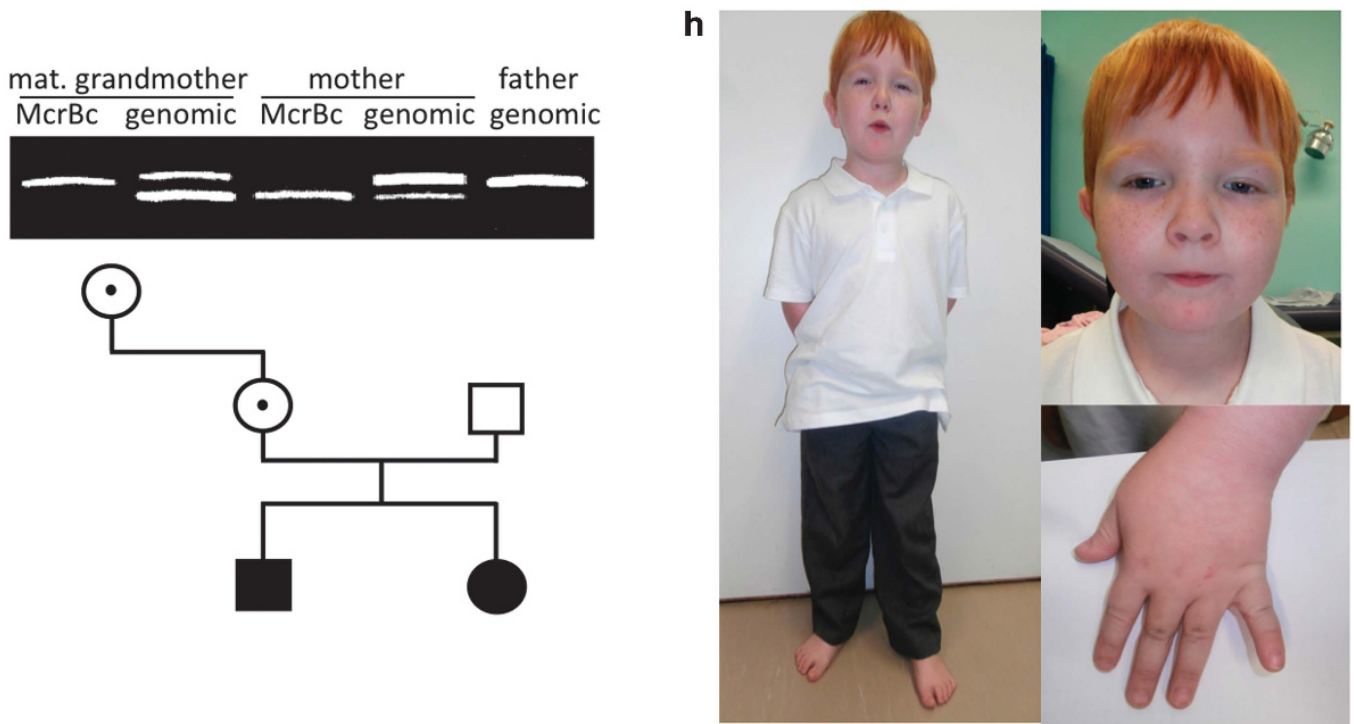
methodological details are available on request, and also the bed file of the capture array. Sequences were visualised on the Integrative Genomics Viewer v1.4 (www.broadinsitute.org/igv). Raw sequence data are available on request.

Long-range PCR and NGS. Genomic sequences: chr20.GRCh37:g.57413337 57418468 and chr20.GRCh37:g.57416569_57419096 (primers 5'-GCCCATC ATTTGATTTTCTAGGGCCAAG-3'; $5^{\prime}$-GGAGCTGAGTACCAGTCTCTCA GGCAG-3' $5132 \mathrm{bp}$ and $5^{\prime}$-GCGCCAGTGCCTCCAGCTGCCG-3'; 5'-CCTT CCACACAGCTGCAGAAAATGAAG-3'2529 bp) were amplified in individuals with PHP1b. Sequencing libraries were prepared and indexed using the Nextera DNA sample preparation kit and Nextera indexing kit (Illumina UK) following the manufacturer's protocols, and sequenced using the MiSeq micro reagent kit, 500 cycles (Illumina UK).

Sanger sequencing. The deletions in family 1 (chr20.GRCh37:g.57416653 57416693del) and family 2 (chr20.GRCh37:g.57418256_57418290del) were amplified using primers 5'-GCGCCAGTGCCTCCAGCTGCCG-3'; 5'-CGTT CAACCCTGGTAGCCCGTAGGG-3' $(220 \mathrm{bp})$ and $5^{\prime}$-CCATGTTCACATGTA GCGAGGAGGG-3'; 5'-CGGGGGTTGGTATAGCTCTCAGTTGC-3' (273 bp) and verified by Sanger sequencing. To determine inheritance in the mother and maternal grandmother, $200 \mathrm{ng}$ genomic DNA was amplified after restriction digestion with restriction enzyme Mcrbc (New England Biolabs UK, Hitchin, UK) according to the manufacturer's instructions, as described. ${ }^{15}$

\section{Bioinformatic analysis}

We developed a bioinformatic pipeline for identifying both single-nucleotide variants (SNVs) and copy number variants (CNVs). Sequence output from targeted NGS was aligned to a human reference sequence (UCSC Genome Browser hg19, http://hgdownload.cse.ucsc.edu/goldenpath/hg19/chromosomes/) using Burrows-Wheeler Aligner (BWA-MEM-version 0.7.5a) (http://bio-bwa. sourceforge.net) to produce BAM files (binary version of Sequence Alignment/ Map Format). Duplicate reads were removed from the BAM files using Picard tools (version 1.95) (http://picard.sourceforge.net) to avoid biases that might skew variant predictions. Local indel realignment was undertaken by the 'Indel Realigner' function from the Genome Analysis Toolkit (GATK-version 2.4-9 ${ }^{16}$ ) to avoid misalignment that might cause mismatches and compromise base quality recalibration. Base quality recalibration was done using GATK 'Table Recalibration' function, which analysed several covariates to remove systematic biases and improve accuracy of quality scores. ${ }^{17}$ All produced aligned files were further used to calculate average read depth in $1000 \mathrm{bps}$ non-overlapping windows within the captured region (Supplementary Table 2). The BAM files were also used to normalise read depth between samples, which was used to compare samples between different pools (Supplementary Table 2). These realigned and recalibrated BAM files were then used to determine SNVs and CNVs. Variant (SNVs eg SNPs and indel alleles) predictions and genotyping were performed using the GATK 'Unified Genotype' function for each sample by computing allele frequency distribution, ${ }^{18}$ and raw variant calls were outputted in VCF (variant call format). An individual variant filtration (such as Quality by Depth $<2.0$, Fisher Strand Bias $>60.0$ etc) was performed for both SNPs and indels using GATK Variant Filtration method to remove low quality and potentially false positive sites. Variant data were further annotated by Annovar to provide functional annotation of variants. ${ }^{19}$

Pindel (version $0.2 .4^{18}$ ), a structural variant detection tool that can detect breakpoints of large deletions and medium-sized insertions from paired-end short reads, was used to perform $\mathrm{CNV}$ predictions. These $\mathrm{CNV}$ s were further filtered based on variant size, alternate allele frequency, CA/GT homopolymer repeats and known SNPs. These filters increased sensitivity and specificity of the predictions, which reduced false negatives and false positives.

The variants identified in Patients 1, 2 and 9 have been submitted to LOVD (www.lovd.nl/GNAS variants GNAS_00174, GNAS_00175, GNAS_00176).

\section{RESULTS}

In seven PHP1b patients, the GNAS locus (chr20.GRCh37: g.57200800_57627000) was enriched by targeted capture, and analysed by next-generation sequencing. On average, more than $98 \%$ of the captured regions had read depth greater than 10, showing that the sequence data were of high quality in our region of interest (Supplementary Table 3). Case 1 of family 1, one of two affected siblings, harboured a 40-bp deletion spanning the genomic co-ordinates chr20.GRCh37:g.57416653_57416693del (family 1, Figure 1c). This deletion was intronic to both NESP55 and NESPAS. It was not present in dbSNP137, Repeat Masker (http:// genome.ucsc.edu) or in 168 control samples. The deletion was detected in the affected sibling and also in the siblings' mother who showed no clinical signs of PHP1b. To determine the parental origin of the deletion in the mother, her DNA was amplified after digestion with methylation-specific restriction enzymes Bst $\mathrm{UI}$ and $\mathrm{McrBc}$ (which digest only unmethylated and methylated DNA, respectively). The deletion was detectable only in DNA restricted by $\mathrm{McrBc}$, demonstrating that it was present on her unmethylated, maternally inherited allele (Figure 1d and g). The maternal grandmother of the affected sibs also harboured the deletion, though methylation-specific restriction analysis in her case showed it to be on the paternally inherited allele (Figure 1g).

Building on this finding, the genomic region chr20.GRCh37: g.57413337_57419096 was amplified by long-range PCR and sequenced by NGS in samples from an additional 14 PHP1b patients with no known underlying genetic aberration. A 33-bp intronic deletion was identified (chr20.GRCh37:g.57418256_57418290del) in one further patient (case 9, Figure 1, Table 1). Targeted sequencing showed the same deletion to be present in his unaffected mother. The deletion was too distant from the DMR to use methylation-specific restriction to determine its origin in the mother. The deletion was not present in the maternal grandmother of patient 2 (data not shown); the maternal grandfather was deceased and no DNA was available for analysis, but he was not known to have any clinical features of PHP1b. The variant in

Figure 1 Identification of small GNASAS deletions in PHP1b patients. (a) Schematic of normal gene transcription and differential methylation within the GNAS cluster. Red and blue indicate maternal and paternal transcripts, respectively. Open and closed boxes represent non-coding and coding transcripts, respectively, while dotted lines indicate splicing. Black 'lollipops' indicate locations of differential methylation, which is on the maternally derived allele for the GNASAS, GNAS-XL and GNAS A/B DMRs and the paternally derived allele for the NESP55 DMR. (b) UCSC screenshot (GRCh37/hg19) of GNAS-imprinted region. Green boxes indicate $\mathrm{CpG}$ islands and blue rectangles linked by arrowed lines indicate positions of transcripts within the locus. Below the screenshot, blue lines illustrate deletions associated with PHP1b identified by $(1,2)$ Bastepe et al, ${ }^{9}(3)$ Chillambhi et al ${ }^{10}$ and $(4)$ Richard et al. ${ }^{11}$ Red lines indicate positions of deletions in families 1 and 2. (c-e): the deletion in family 1. (c) Screenshot from IGV illustrating the deletion in case 1, family 1-note the black lines indicating deletion of nucleotides, and the reduction in overall sequence depth. (d) Sanger sequencing electropherogram of the same region in the mother of family 1, after restriction of genomic DNA with McrBc, which cleaves methylated DNA. Only the deleted allele remains, indicating that this deleted sequence is present on her paternally inherited allele. (e) Agarose gel electrophoresis of DNA from the parents and maternal grandmother in family 1 , with and without restriction by McrBc to digest unmethylated DNA. This shows in the maternal grandmother that the deletion is digested, ie, present on the unmethylated paternal allele. In contrast, the deletion is on the maternal, unmethylated allele in the mother and absent from the unrelated father. (f-h) The deletion in case 9, family 8. (f) Screenshot from IGV illustrating the deletion in proband 2-note the black lines indicating deletion of nucleotides, and the reduction in overall sequence depth. (g) Sanger sequencing electropherogram of genomic DNA in proband 2, showing disruption of normal sequence originating at the deletion. (h) Clinical photographs of case 9. Note some facial features of PHPla including flattening of nasal bridge, anteverted nares and small hands. 
family 2 represented a deletion of two of three copies of an 18-bp repeat element; deletion of one copy was represented within dbSNP137 as rs36230182 (minor allele frequency unknown); however, there was no evidence of any deletion in 168 normal controls. In the remaining 13 patients, CNVs were called by Pindel and SNPs were called by GATK, but no novel variants were identified (data not shown).

DNA methylation analysis showed the affected sibs in family 1 had essentially complete paternalisation of the GNAS cluster, while hypomethylation in proband 2 was incomplete, except at the GNAS A/B DMR (Supplementary Table 1).

\section{DISCUSSION}

Like other imprinting disorders, PHP1b is clinically and molecularly heterogeneous. Sporadic PHP1b is associated with UPD20pat and epimutation of DMRs throughout the GNAS cluster; most heritable PHP1b results from epimutation of the GNAS $A / B$ DMR-associated with maternal STX16 deletions-and rarely from deletions of NESP55 of maternal origin. We used NGS of the GNAS locus to seek genetic lesions underlying epimutations in PHP1b patients. In two affected siblings, a variant was found in the first intron of NESP55, within the 3-kb deletion described by Bastepe et al. ${ }^{9}$ Further localised sequencing within this region revealed another deletion in a third, unrelated individual. Both variants were maternally transmitted, but the mothers showed no clinical or epigenetic evidence of PHP1b. In family 1 , the clinically unaffected mother inherited the deletion from her own mother, showing that maternal inheritance of this deletion does not always cause PHP1b.

Large NESP55 deletions are believed to abrogate germline transcription from the NESP promoter, resulting in aberrant methylation setting throughout the whole imprinted cluster. ${ }^{9,12}$ Notably, the variants reported here are close to the transcriptional start site of NESP55, overlapping those described by Bastepe, Chillambhi and Richard et al. ${ }^{9-11}$ Such deletions potentially delineate critical sequences for imprint setting of this locus.

We detected variants in only two of 20 PHP1b index cases (albeit with different extents of sequencing). NESP deletions $>1 \mathrm{~kb}$ were not detected in any individual. By contrast, STX16 deletions are found in essentially all individuals with GNAS $A / B$ hypomethylation. It remains unclear whether genetic NESP mutations are uncommon in PHP1b. It may be that in the patients we studied, some variants remained undetected because of discontinuities in sequence coverage; CNVs (like the STX16 deletion) may arise between repetitive sequences, but these sequences cannot be unambiguously captured, results in reduced or absent coverage. Further variants may lie outside the region we targeted, in more distant chromatin-regulating domains. Alternatively, it may be that the majority of PHP1b is indeed sporadic, caused by epimutations without direct genetic cause. Genetic mutations underlying epimutations have been reported very infrequently in other imprinting disorders, including Russell-Silver Syndrome, transient neonatal diabetes mellitus and Beckwith-Wiedemann syndrome associated with KCNQ1OT1 (imprinting control region 2) hypomethylation. However, a significant minority of BWS patients with H19 (ICR1) hypermethylation have maternally inherited deletions or point mutations of ICR $1 .{ }^{20}$ Genomic analysis of other patient groups could potentially identify mutations that would help to uncover further determinants of epigenetic function.

It is striking that the individuals with NESP55 deletions were not identical, either in phenotype or in DNA methylation status. Clinical features in the affected individuals were somewhat atypical of PHP1b, which is associated chiefly with biochemical features of parathormone resistance in the absence of dysmorphism. ${ }^{3}$ The probands of family 1 have mild learning difficulties, and one has calcifications in brain tissue; the proband in family 2 has educational difficulties warranting educational support, and has short stature, which is more typical of PHP1a. It is recognised that a minority of individuals with PHP1b epimutations have clinical features overlapping with PHP1a. ${ }^{21-24}$ In the three individuals with PHP1b, the deletions resulted in epimutations throughout the GNAS cluster; but while the siblings in family 1 had almost complete hypomethylation, the proband in family 2 had less marked hypomethylation, except at the GNAS A/B DMR. In family 1 , a deletion was transmitted maternally through three generations, but only the two-third-generation cases 1 and 2 presented with PHP1b. This may be because the deletion is coincidental to PHP-though given the presence of deletions in two of 20 pedigrees, and their absence from controls and databases, this seems unlikely. It may be that these small deletions are not fully penetrant, and do not always cause PHP1b. Alternatively, the severity of the deletion's effect may increase with successive generations, causing apparent genetic anticipation-this phenomenon has been observed in maternally transmitted BWS associated with hypermethylation of H19. ${ }^{15}$ Investigation of further cases will shed light on these unresolved issues. In summary, genomic sequencing is a powerful tool with potential to reveal non-coding genetic variants that may underlie epigenetic disorders.

\section{CONFLICT OF INTEREST}

The authors declare no conflict of interest.

\section{ACKNOWLEDGEMENTS}

We are grateful to the patients and families who provided the samples for this study, and also the referring clinicians. RLP was funded by the Newlife Foundation for Disabled Children (Grant 09-10/07) and FIR by the Medical Research Council (MR/J000329/1). The research was supported by the Hampshire and Isle of Wight NIHR Comprehensive Local Research Network.

1 Bastepe M: Genetics and epigenetics of parathyroid hormone resistance. Endocr Dev 2013; 24: 11-24.

2 Mantovani G: Clinical review: pseudohypoparathyroidism: diagnosis and treatment J Clin Endocrinol Metab 2011; 96: 3020-3030.

3 Kelsey G: Imprinting on chromosome 20: tissue-specific imprinting and imprinting mutations in the GNAS locus. Am J Med Genet C Semin Med Genet 2010; 154C 377-386.

4 Liu J, Litman D, Rosenberg MJ, Yu S, Biesecker LG, Weinstein LS: A GNAS imprinting defect in pseudohypoparathyroidism type IB. J Clin Invest 2000; 106 1167-1174.

5 Maupetit-Méhouas S, Mariot V, Reynès $\mathrm{C}$ et al: Quantification of the methylation at the GNAS locus identifies subtypes of sporadic pseudohypoparathyroidism type Ib. J Med Genet 2011; 48: 55-63.

6 Mantovani G, Linglart A, Garin I, Silve C, Elli FM, de Nanclares GP: Clinical utility gene card for: pseudohypoparathyroidism. Eur J Hum Genet 2013; 21: 6.

7 Bastepe M, Fröhlich LF, Hendy GN et al: Autosomal dominant pseudohypoparathyroidism type $\mathrm{Ib}$ is associated with a heterozygous microdeletion that likely disrupts a putative imprinting control element of GNAS. J Clin Invest 2003; 112: $1255-1263$.

8 Linglart A, Gensure RC, Olney RC, Jüppner H, Bastepe M: A novel STX16 deletion in autosomal dominant pseudohypoparathyroidism type Ib redefines the boundaries of a cis-acting imprinting control element of GNAS. Am J Hum Genet 2005; 76 804-814.

9 Bastepe M, Fröhlich LF, Linglart A et al: Deletion of the NESP55 differentially methylated region causes loss of maternal GNAS imprints and pseudohypoparathyroidism ibe Nat Genet 2005; 37: 25-27.

10 Chillambhi S, Turan S, Hwang DY, Chen HC, Jüppner H, Bastepe M: Deletion of the noncoding GNAS antisense transcript causes pseudohypoparathyroidism type Ib and biparental defects of GNAS methylation in cis. J Clin Endocrinol Metab 2010; 95 3993-4002. 
11 Richard N, Abeguilé G, Coudray N et al: A new deletion ablating NESP55 causes loss of maternal imprint of A/B GNAS and autosomal dominant pseudohypoparathyroidism type Ib. J Clin Endocrinol Metab 2012; 97: E863-E867.

12 Chotalia M, Smallwood SA, Ruf N et al: Transcription is required for establishment of germline methylation marks at imprinted genes. Genes Dev 2009; 23 105-117.

13 Bastepe M, Lane AH, Jüppner $H$ : Paternal uniparental isodisomy of chromosome $20 \mathrm{q}$ - and the resulting changes in GNAS1 methylation-as a plausible cause of pseudohypoparathyroidism. Am J Hum Genet 2001; 68: 1283-1289.

14 Poole RL, Docherty LE, Al Sayegh A et al: International Clinical Imprinting Consortium. Targeted methylation testing of a patient cohort broadens the epigenetic and clinical description of imprinting disorders. Am J Med Genet A 2013; 161: 2174-2182.

15 Berland S, Appelbäck M, Bruland $O$ et al: Evidence for anticipation in BeckwithWiedemann syndrome. Eur J Hum Genet 2013; 21: 1344-1348.

16 McKenna A, Hanna M, Banks E et al: The Genome Analysis Toolkit: a MapReduce framework for analyzing next-generation DNA sequencing data. Genome Res 2010; 20: 1297-1303.

17 DePristo M, Banks E, Poplin R et al: A framework for variation discovery and genotyping using next-generation DNA sequencing data. Nat Genet 2011; 43 491-498.

18 Wang K, Li M, Hakonarson H: ANNOVAR: functional annotation of genetic variants from next-generation sequencing data. Nucleic Acids Res 2010; 38: e164.

19 Ye K, Schulz MH, Long Q, Apweiler R, Ning Z: Pindel: a pattern growth approach to detect break points of large deletions and medium sized insertions from paired-end short reads. Bioinformatics 2009; 25: 2865-2871.

20 Beygo J, Citro V, Sparago A et al: The molecular function and clinical phenotype of partial deletions of the IGF2/H19 imprinting control region depends on the spatial arrangement of the remaining CTCF-binding sites. Hum Mol Genet 2013; 22: 544-557.

21 de Nanclares GP, Fernández-Rebollo E, Santin I et al: Epigenetic defects of GNAS in patients with pseudohypoparathyroidism and mild features of Albright's hereditary osteodystrophy. J Clin Endocrinol Metab 2007; 92: 2370-2373.

22 Mariot V, Maupetit-Méhouas S, Sinding C, Kottler ML, Linglart A: A materna epimutation of GNAS leads to Albright osteodystrophy and parathyroid hormone resistance. J Clin Endocrinol Metab 2008; 93: 661-665.

23 Mantovani G, de Sanctis L, Barbieri AM et al: Pseudohypoparathyroidism and GNAS epigenetic defects: clinical evaluation of Albright hereditary osteodystrophy and molecular analysis in 40 patients. J Clin Endocrinol Metab 2010; 95: 651-658.

24 Unluturk U, Harmanci A, Babaoglu M et al: Molecular diagnosis and clinical characterization of pseudohypoparathyroidism type-lb in a patient with mild Albright's hereditary osteodystrophy-like features, epileptic seizures, and defective renal handling of uric acid. Am J Med Sci 2008; 336: 84-90.

(c) (i) This work is licensed under a Creative Commons Attribution 3.0 Unported License. The images or other third party material in this article are included in the article's Creative Commons license, unless indicated otherwise in the credit line; if the material is not included under the Creative Commons license, users will need to obtain permission from the license holder to reproduce the material. To view a copy of this license, visit http:// creativecommons.org/licenses/by/3.0/

Supplementary Information accompanies this paper on European Journal of Human Genetics website (http://www.nature.com/ejhg) 